Revista Brasileira de Agricultura Irrigada v.14, nº.3, p. 4029 - 4036, 2020

ISSN 1982-7679 (On-line)

Fortaleza, CE, INOVAGRI - http://www.inovagri.org.br

DOI: $10.7127 /$ rbai.v14n101132

Protocolo 1132.20 - 01/04/2020 Aprovado em 29/10/2020

\title{
APROVEITAMENTO DE ÁGUAS PLUVIAIS PARA IRRIGAÇÃO DE HORTA NO MUNICÍPIO DE CAMPO NOVO DO PARECIS - MT
}

\author{
Acácio Perboni ${ }^{1}$, Líssia Letícia de Paiva Oliveira ${ }^{2}$, Éliton dos Santos ${ }^{3}$, Leonardo Jardim de Godoy ${ }^{3}$, \\ Luiz Henrique Demenighi ${ }^{3}$
}

\begin{abstract}
RESUMO
Devido à escassez ou má distribuição da chuva em algumas localidades, técnicas alternativas para obtenção de água devem ser estudadas. O objetivo deste trabalho foi avaliar a viabilidade de suprir com a captação de águas pluviais, toda a demanda de irrigação do setor de horta do Campus Campo Novo do Parecis, do Instituto Federal de Educação, Ciência e Tecnologia de Mato Grosso. A quantidade mensal de água para irrigar o setor de horta, foi obtida com o balanço hídrico, utilizando os valores de precipitação, evapotranspiração de referência da cultura e capacidade de água disponível no solo. O período de maior deficiência hídrica na região ocorre na época seca, entre os meses de maio a setembro. Já o período de menores demandas de água necessária para irrigação, ocorre entre os meses de outubro e abril, na época das chuvas. Para irrigar a área de $2.080 \mathrm{~m}^{2}$ do setor de horta com o aproveitamento de águas pluviais, captando água da chuva em $1.922 \mathrm{~m}^{2}$ de área de telhado é necessário reservatório com capacidade de $1.402 \mathrm{~m}^{3}$.
\end{abstract}

Palavras chaves: chuva, reservatório, recursos hídricos.

\section{USE OF RAINWATER FOR VEGETABLE GARDEN IRRIGATION IN THE CITY OF CAMPO NOVO DO PARECIS - MT}

\begin{abstract}
Due to the scarcity or poor distribution of rain in some locations, alternative techniques for obtaining water should be studied. The objective of this work was to evaluate the feasibility of supplying with rainwater harvesting, all the irrigation demand of the vegetable garden sector of the Campo Novo do Parecis Campus, from the Federal Institute of Education, Science and Technology of Mato Grosso. The monthly amount of water to irrigate the vegetable garden sector was obtained from the water

\footnotetext{
${ }^{1}$ Doutor em Engenharia de Sistemas Agrícolas e Docente do Instituto Federal do Mato Grosso, MT 235 Km 12 , s/n, Campo Novo do Parecis, MT, Brasil, CEP: 78360-000. E-mail: acacio_perboni@ yahoo.com.br

2 Doutora em Manejo de Solo e Água, Universidade Federal Rural do Semi-Árido, Rua Francisco Mota Bairro, 572, Presidente Costa e Silva, Mossoró, RN, Brasil, CEP: 59625-900. E-mail: leticia_lissia@ hotmail.com

${ }^{3}$ Estudante de Agronomia, Instituto Federal do Mato Grosso, MT $235 \mathrm{Km}$ 12, s/n, Campo Novo do Parecis, MT, Brasil, CEP: 78360-000. E-mail: eliton.santos12@hotmail.com; luizdm10@hotmail.com; leandrojgodoy@hotmail.com
} 
balance, using the values of precipitation, reference evapotranspiration of the crop and available water capacity in the soil. The period of greatest water deficiency in the region occurs in the dry season, between the months of May to September. The period of lowest water demands for irrigation, on the other hand, occurs between the months of October and April, in the wet season. To irrigate the $2,080 \mathrm{~m}^{2}$ area of the vegetable garden with the use of rainwater, capturing rainwater in $1,922 \mathrm{~m}^{2}$ of roof area, a reservoir with a capacity of $1,402 \mathrm{~m}^{3}$ is required.

Keywords: rain, reservoir, water resources.

\section{INTRODUÇÃO}

No Brasil estima-se que a agricultura irrigada seja responsável pelo uso consuntivo de $46 \%$ e consumo de $67 \%$ de toda a água retirada do meio ambiente (AGÊNCIA NACIONAL DAS ÁGUAS, 2017).

Segundo Bezerra et al. (2010) o aproveitamento de água de chuva pode reduzir o uso de recursos hídricos de qualidade superior e prevenir a escassez da água potável. A água de chuva pode ser aproveitada em atividades que não necessitam água potável, por exemplo, na descarga de bacias sanitárias, na irrigação e na limpeza de pisos, equipamentos e veículos (GIL et al., 2020; NOGUEIRA et al., 2016; TEIXEIRA et al., 2016). Em regiões onde se justifica a necessidade, a água de chuva é aproveitada para fins potáveis (NEU et al., 2018).

Para o armazenamento da água pluvial é necessário à construção de reservatório, com a função de reter e acumular a água captada, para posterior utilização. Gheyi et al. (2012) relatam sobre a importância do armazenamento das águas pluviais para uso agrícola, considerando uma alternativa viável para criação de animais e produção de alimentos.

Botari e Prado (2018) dimensionaram reservatório para aproveitamento da água da chuva na irrigação de um espaço público aberto na cidade de Umuarama - PR. Os autores verificaram que apesar da precipitação anual média do município ser $1.482 \mathrm{~mm}$ é necessário reservatório, devido à distribuição desuniforme das chuvas.

No Campus Campo Novo do Parecis do Instituto Federal de Educação, Ciência e Tecnologia de Mato Grosso (IFMT), é utilizada água de poço profundo para irrigação do setor de horta. A irrigação é realizada para suprir toda a demanda do setor na época seca, e de forma complementar a chuva na época das chuvas. Aumentando desta forma os custos com energia elétrica para bombear a água até a superfície, e o consumo hídrico de água potável.

Diante do exposto, o objetivo deste trabalho foi avaliar a viabilidade de suprir com captação de águas pluviais, toda a demanda de irrigação do setor de horta do Campus Campo Novo do Parecis do IFMT.

\section{MATERIAL E MÉTODOS}

O estudo foi realizado no Campus Campo Novo do Parecis, do Instituto Federal de Mato Grosso (IFMT), com coordenadas geográficas $13^{\circ} 40^{\prime} 31^{\prime \prime} \mathrm{S}$ e $57^{\circ} 53^{\prime} 31^{\prime \prime} \mathrm{W}$, e altitude média de $574 \mathrm{~m}$, localizado no município de Campo Novo do Parecis - MT. O clima local é do tipo Aw conforme classificação de Köppen, que significa clima tropical com seca no inverno e chuva no verão. O solo da área em estudo é classificado como Latossolo Vermelho distrófico típico (DALCHIAVON et al., 2015), com relevo suave ondulado e boa drenagem.

A pesquisa foi desenvolvida em duas etapas. $\mathrm{Na}$ primeira foi determinada a necessidade de água para irrigação do setor de horta. E na segunda etapa, foi realizado dimensionamento do reservatório para armazenamento de água da chuva.

As áreas utilizadas para produção no setor de horta (Figura 1) foram medidas utilizando trena de 50 metros, obtendo-se área total de $2.080 \mathrm{~m}^{2}$. As principais culturas cultivadas no setor são alface, almeirão, cebolinha, quiabo, maxixe, coentro e rúcula. 


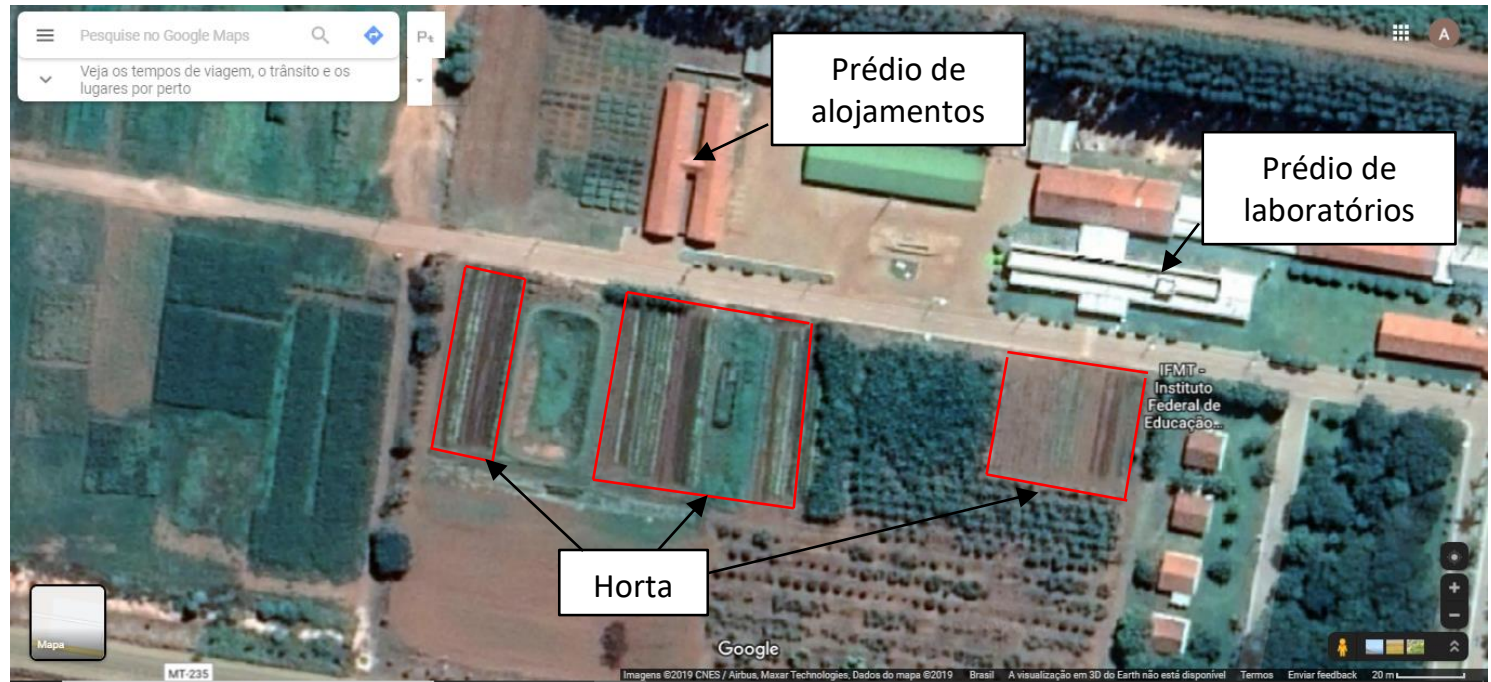

Figura 1. Localização do setor de horta e dos prédios para captação de água da chuva. Fonte da imagem: Google.

Para obter o volume de água da chuva possível de ser captado, foram considerados os telhados dos prédios de laboratórios e de alojamentos, indicados na Figura 1. Foram observadas áreas de telhados de $884 \mathrm{~m}^{2}$ na planta de cobertura do prédio de laboratórios e $1.038 \mathrm{~m}^{2}$ no prédio de alojamento dos alunos internos, em medição com trena, totalizando área de captação de água da chuva de 1.922 $\mathrm{m}^{2}$.

\section{Estimativa da necessidade de água para irrigação do setor de horta}

Inicialmente foi utilizada a equação de Penman Monteith (equação 1) para calcular a evapotranspiração de referência (ETo) média mensal (ALLEN et al., 2006).

$\mathrm{ETo}=\frac{\left[0,408^{*} \mathrm{~s}^{*}(\mathrm{Rn}-\mathrm{G})+\gamma^{*} \frac{900}{(\mathrm{~T}+273)} * \mathrm{~V}_{2 \mathrm{~m}}{ }^{*} \Delta_{\mathrm{e}}\right]}{\left[\mathrm{s}+\mathrm{y}^{*}\left(1+0,34^{*} \mathrm{~V}_{2 \mathrm{~m}}\right)\right]}$

Em que: s é a declividade da curva de saturação no gráfico psicrométrico $\left(\mathrm{kPa} .{ }^{\circ} \mathrm{C}^{-1}\right)$; $\mathrm{Rn}$ é a radiação líquida total diária $\left(\mathrm{MJ} \cdot \mathrm{m}^{-2} \cdot \mathrm{d}^{-}\right.$ ${ }^{1}$ ); G é o fluxo de calor no solo $\left(\mathrm{MJ} . \mathrm{m}^{-2} \cdot \mathrm{d}^{-1}\right) ; \gamma$ é a constante psicrométrica $\left(\mathrm{kPa}^{\circ} \mathrm{C}^{-1}\right)$; $\mathrm{T}$ é a temperatura média $\left({ }^{\circ} \mathrm{C}\right) ; \mathrm{V}_{2} \mathrm{~m}$ é a velocidade do vento a $2 \mathrm{~m}\left(\mathrm{~m} . \mathrm{s}^{-1}\right)$; e $\Delta$ e é inclinação da curva de pressão de vapor $\left(\mathrm{kPa}^{\circ} \mathrm{C}^{-1}\right)$.

Os dados climáticos necessários para o cálculo da ETo, foram obtidos do Instituto Nacional de Meteorologia - INMET, de estação meteorológica automática localizada a uma distância em linha reta de $13,4 \mathrm{~km}$ do setor de horta, na Cooperativa Agrícola de Produtores de Cana de Campo Novo do Parecis - COOPRODIA.

A série histórica disponibilizada pelo INMET iniciou em 2002. Foi verificado grande quantidade de dados faltantes, e optouse por analisar as observações referentes aos anos de 2012, 2013, 2015, 2016 e 2018. Nestes, a pequena quantidade de dados faltantes, foram preenchidos com a média das observações mais próximas. E finalmente foram obtidas as médias mensais dos elementos climáticos, com os dados diários.

$\mathrm{Na}$ sequência foi calculada a evapotranspiração da cultura sob irrigação localizada $\left(\mathrm{ETC}_{\mathrm{Loc}}\right)$ com a equação 2, para estimar a necessidade de água das culturas ao longo do ciclo.

$$
\mathrm{ETc}_{\mathrm{Loc}}=\mathrm{ETo} * \mathrm{Kc} * \mathrm{~K}_{\mathrm{L}}
$$

Em que: ETo é a evapotranspiração de referência $\left(\mathrm{mm} \cdot \mathrm{d}^{-1}\right)$; Kc é o coeficiente de cultivo; $K_{L}$ é o fator de correção devido a localização da água aplicada.

Adotou-se o valor médio de Kc igual a 1,0 para hortaliças, de acordo com o boletim 56 da FAO (ALLEN et al., 2006).

Considerou-se o valor de $\mathrm{K}_{\mathrm{L}}$ igual a 1,0 conforme o método de Fereres (1981), o qual determina que se $\mathrm{P} \geq 65 \% \rightarrow \mathrm{K}_{\mathrm{L}}=1$, em que $\mathrm{P}$ é a porcentagem da área sombreada (PAS) ou molhada 
(PAM), prevalecendo o maior valor (MANTOVANI et al., 2009).

O balanço hídrico para controle da irrigação em escala mensal foi realizado em planilha do software Microsoft Excel, utilizando os valores de precipitação $(\mathrm{P})$, evapotranspiração de referência da cultura sob irrigação localizada $\left(\mathrm{ETC}_{\text {Loc }}\right)$ e capacidade de água disponível no solo (CAD). Este método é uma modificação do balanço hídrico climatológico sequencial de Thornthwaite e Mather (1955), no qual é considerado o intervalo de umidade do solo em que a cultura consegue extrair água na mesma taxa da evapotranspiração.

A CAD foi calculada com a equação 3 .

$\mathrm{CAD}=\frac{\left(\theta_{\mathrm{CC}}-\theta_{\mathrm{PMP}}\right)}{10} * \mathrm{Z}$

Em que: $\theta_{\text {CC }}$ é a umidade à base de volume na capacidade de campo $\left(\mathrm{cm}^{3} . \mathrm{cm}^{-3}\right) ; \theta_{\mathrm{PMP}}$ é a umidade à base de volume no ponto de murcha permanente $\left(\mathrm{cm}^{3} \cdot \mathrm{cm}^{-3}\right)$; $\mathrm{Z}$ é a profundidade efetiva do sistema radicular $(\mathrm{cm})$.

A profundidade efetiva do sistema radicular utilizada para determinação da CAD foi de $20 \mathrm{~cm}$, conforme recomendações do boletim 56 da FAO, para hortaliças (ALLEN et al., 2006).

Para obtenção dos valores de $\theta_{\mathrm{CC}}$ e $\theta_{\mathrm{PMP}}$, foram coletas seis amostras indeformadas de solo, três na camada de 0 a $10 \mathrm{~cm}$ e três na camada de 10 a $20 \mathrm{~cm}$, utilizando trado tipo "castelinho" e anéis com dimensões de $5 \mathrm{~cm}$ de altura e $5 \mathrm{~cm}$ de diâmetro. Após a coleta, os anéis foram revestidos com papel laminado, embalados e encaminhados ao Laboratório de Solos e Qualidade de Água, da Escola Superior de Agricultura "Luiz de Queiroz" da Universidade de São Paulo. No qual foi elaborada a curva de retenção, aplicando tensões de 0,$1 ; 0,3 ; 0,5 ; 1 ; 5$ e 15 bar.

$\mathrm{O}$ valor de umidade na capacidade de campo foi determinado na curva de retenção, na tensão de 0,3 bar, que representa a condição de solos argilosos. E a umidade no ponto de murcha permanente, na tensão de 15 bar.

A CAD é a água disponível entre a umidade na capacidade de campo $\left(\theta_{\mathrm{CC}}\right)$ e a umidade no ponto de murcha permanente $\left(\theta_{\mathrm{PMP}}\right)$. Para que as plantas não sofram estresse hídrico, é considerado que estas conseguem absorver apenas uma fração da CAD, conhecida como água facilmente disponível (AFD), e calculada com a equação 4.

$$
\mathrm{AFD}=\mathrm{CAD} * \mathrm{f}
$$

Em que: CAD é a capacidade de água disponível no solo $(\mathrm{mm})$; f é fração de água disponível.

De acordo com Mantovani et al. (2009), para hortaliças, o valor da fração de água disponível (f) é 0,5.

$\mathrm{Na}$ elaboração do balanço hídrico, considera-se que no primeiro dia a água facilmente disponível inicial (AFDI) é igual a CAD. E a água facilmente disponível final do dia (AFDF) é calculada, conforme equação 5.

$$
\mathrm{AFDF}=\mathrm{AFDI}+\left(\mathrm{P}+\mathrm{I}-\mathrm{ETC}_{\mathrm{Loc}}\right)
$$

Em que: AFDI é a água facilmente disponível inicial $(\mathrm{mm})$; P é a precipitação $(\mathrm{mm})$; I é a irrigação (mm); ETcLoc é a evapotranspiração da cultura sob irrigação localizada (mm).

A partir do segundo dia a AFDI será igual à AFDF do dia anterior ou igual a CAD quando for realizada irrigação.

Para determinar a lâmina de água aplicada na irrigação (I) utiliza-se a equação 6 .

$\mathrm{I}=\mathrm{CAD}-\mathrm{AFDF}$

Em que: CAD é a água facilmente disponível (mm); e AFDF é a água facilmente disponível final $(\mathrm{mm})$.

A irrigação será calculada apenas quando a AFDF do dia anterior for igual ou inferior a AFD.

Os resultados encontrados através do método descrito acima, e calculados para cada dia, foram agrupados em intervalos mensais e utilizados para elaboração do balanço hídrico para controle da irrigação. Finalmente com os dados mensais de lâmina de irrigação, calculase a demanda mensal de água da horta ( $D_{\text {horta }}$ ), 
utilizando a equação 7.

$$
\mathrm{D}_{\text {horta }}=\frac{\left(\frac{\mathrm{I}}{1.000} * \mathrm{~A}\right)}{\mathrm{Ea}}
$$

Em que: I é a irrigação (mm); A é a área da horta $\left(\mathrm{m}^{2}\right)$; e Ea é a eficiência de aplicação do sistema de irrigação.

A eficiência de aplicação de água (Ea) utilizada foi de $90 \%$, pois a irrigação no setor de horta é realizada com sistemas de irrigação localizada (MANTOVANI et al., 2009).

\section{Dimensionamento da estrutura de armazenamento de água da chuva}

De acordo com Moruzzi et al. (2012), vários métodos para o dimensionamento do reservatório estão disponíveis na literatura. Neste trabalho foi utilizado o método de Rippl (equações 8, 9 e 10), que é um dos métodos indicados pela Associação Brasileira de Normas Técnicas (ABNT), na norma ABNT NBR 15527 de 2007.

$\mathrm{S}_{(\mathrm{t})}=\mathrm{D}_{(\mathrm{t})}-\mathrm{Q}_{(\mathrm{t})}$

$Q_{(t)}=C \times$ precipitação $_{(t)} \times$ área de captação

$V_{0}=\Sigma \mathrm{S}_{(\mathrm{t})}$ somente para valores de $\mathrm{S}_{(\mathrm{t})}>0$
Em que: $\mathrm{S}_{(\mathrm{t})}$ é o volume de água no reservatório no tempo $\mathrm{t}\left(\mathrm{m}^{3}\right) ; \mathrm{D}_{(\mathrm{t})}$ é a demanda ou consumo no tempo $\mathrm{t}\left(\mathrm{m}^{3}\right) ; \mathrm{Q}_{(\mathrm{t})}$ é o volume de chuva aproveitável no tempo $\mathrm{t}\left(\mathrm{m}^{3}\right) ; \mathrm{C}$ é o coeficiente de escoamento superficial; e $V_{0}$ é o volume do reservatório $\left(\mathrm{m}^{3}\right)$.

O volume de água da chuva aproveitável mensalmente é a multiplicação das áreas dos telhados $\left(1.922 \mathrm{~m}^{2}\right)$, a altura mensal da chuva e o coeficiente de escoamento superficial (C), que de acordo com Azevedo Netto et al. (1998) é de 0,95 para telhados.

\section{RESULTADOS E DISCUSSÃO}

Os dados médios da curva de retenção de água no solo para as camadas de 0 a $10 \mathrm{~cm}$ e de 10 a $20 \mathrm{~cm}$ estão apresentados, na Tabela 1.

Os valores médios da umidade do solo para as duas camadas analisadas, são $0,30 \mathrm{~cm}^{3} \cdot \mathrm{cm}^{-3} \mathrm{na}$ capacidade de campo e $0,22 \mathrm{~cm}^{3} \cdot \mathrm{cm}^{-3}$ no ponto de murcha permanente. Com estes valores, obteve-se a capacidade de água disponível no solo (CAD) de $16 \mathrm{~mm}$.

Tabela 1. Umidade do solo à base de volume $(\theta)$ nas camadas de 0 a 10 e de 10 a $20 \mathrm{~cm}$.

\begin{tabular}{cccccccc}
\hline Profundidade & \multicolumn{7}{c}{$\theta\left(\mathrm{cm}^{3}\right.$ de água.cm $\mathrm{cm}^{-3} \mathrm{de}$ solo $)$} \\
\cline { 2 - 8 }$(\mathrm{cm})$ & Saturado & 0,1 bar & 0,3 bar & 0,5 bar & 1 bar & 5 bar & 15 bar \\
\hline $0-10$ & 0,54 & 0,32 & 0,29 & 0,26 & 0,24 & 0,22 & 0,21 \\
$10-20$ & 0,53 & 0,33 & 0,30 & 0,28 & 0,27 & 0,25 & 0,23 \\
\hline
\end{tabular}

Tabela 2. Balanço hídrico para controle da irrigação mensal para o setor de horta.

\begin{tabular}{cccccccccccc}
\hline Mês & $\begin{array}{c}\mathrm{P} \\
(\mathrm{mm})\end{array}$ & $\begin{array}{c}\text { ETo } \\
(\mathrm{mm})\end{array}$ & $\begin{array}{c}\text { ETc } \\
(\mathrm{mm})\end{array}$ & $\begin{array}{c}\theta_{\mathrm{CC}} \\
\left(\mathrm{cm}^{3} \cdot \mathrm{cm}^{-3}\right)\end{array}$ & $\begin{array}{c}\theta_{\text {PMP }} \\
\left(\mathrm{cm}^{3} \cdot \mathrm{cm}^{-3}\right)\end{array}$ & $\begin{array}{c}\text { CAD } \\
(\mathrm{mm})\end{array}$ & $\begin{array}{c}\text { AFD } \\
(\mathrm{mm})\end{array}$ & $\begin{array}{c}\text { AFDI } \\
(\mathrm{mm})\end{array}$ & $\begin{array}{c}\text { AFDF } \\
(\mathrm{mm})\end{array}$ & $\begin{array}{c}\mathrm{I} \\
(\mathrm{mm})\end{array}$ & $\begin{array}{c}\mathrm{D}_{(\mathrm{t})} \\
\left(\mathrm{m}^{3}\right)\end{array}$ \\
\hline Jan & 240 & 176 & 176 & 0,3 & 0,22 & 16 & 8 & 441 & 505 & 40 & 92 \\
Fev & 180 & 159 & 159 & 0,3 & 0,22 & 16 & 8 & 388 & 410 & 32 & 74 \\
Mar & 216 & 165 & 165 & 0,3 & 0,22 & 16 & 8 & 447 & 498 & 18 & 42 \\
Abr & 98 & 156 & 156 & 0,3 & 0,22 & 16 & 8 & 398 & 340 & 71 & 165 \\
Mai & 33 & 146 & 146 & 0,3 & 0,22 & 16 & 8 & 412 & 298 & 123 & 283 \\
Jun & 37 & 133 & 133 & 0,3 & 0,22 & 16 & 8 & 401 & 305 & 112 & 258 \\
Jul & 9 & 167 & 167 & 0,3 & 0,22 & 16 & 8 & 418 & 260 & 158 & 366 \\
Ago & 4 & 193 & 193 & 0,3 & 0,22 & 16 & 8 & 408 & 219 & 181 & 419 \\
Set & 55 & 191 & 191 & 0,3 & 0,22 & 16 & 8 & 401 & 265 & 143 & 331 \\
Out & 159 & 187 & 187 & 0,3 & 0,22 & 16 & 8 & 422 & 395 & 68 & 157 \\
Nov & 230 & 168 & 168 & 0,3 & 0,22 & 16 & 8 & 426 & 487 & 18 & 42 \\
Dez & 164 & 183 & 183 & 0,3 & 0,22 & 16 & 8 & 429 & 410 & 70 \\
\hline
\end{tabular}

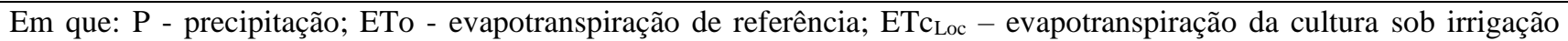
localizada; $\theta \mathrm{cc}$ - umidade na capacidade de campo; $\theta_{\mathrm{PMP}}$ - umidade no ponto de murcha permanente; CAD - capacidade de água disponível; AFD - água facilmente disponível; ADI - água facilmente disponível inicial; ADF - água facilmente disponível final; e I - irrigação; e $\mathrm{D}_{(\mathrm{t})}$ - demanda da horta. 
O balanço hídrico para controle da irrigação em escala mensal para o setor de horta, utilizado para estimar a necessidade de água das culturas, pode ser verificado na Tabela

A água facilmente disponível (AFD) nos meses analisados foi de $8,0 \mathrm{~mm}$. Na penúltima coluna da Tabela 2, são apresentados os valores da soma de todas as lâminas diárias de irrigação para cada mês. E na última coluna os dados de demanda de água para irrigar a horta em cada mês.

O período de maior deficiência hídrica na região em estudo ocorre entre os meses de maio a setembro. Já o período de menores demandas de água necessária para irrigação ocorre entre os meses de outubro e abril. No mês de agosto são necessários $419 \mathrm{~m}^{3}$ de água para irrigar a horta e nos meses de março e novembro apenas $42 \mathrm{~m}^{3}$.
Mossini Junior et al. (2016) realizaram estudos nas cidades de Cáceres, Sapezal e Nova Mutum, todas localizadas no estado do Mato Grosso, sobre a distribuição da precipitação e observaram também que ambas apresentam as estações secas e chuvosas bem definidas ao longo do ano. A distribuição da precipitação na região proporciona o armazenamento de água da chuva na época das chuvas para posterior utilização na época seca.

Após obter os volumes de água para irrigar a horta em cada mês do ano, foi realizado o dimensionamento do volume do reservatório $\left(1.402 \mathrm{~m}^{3}\right)$, somando todos os valores positivos de volume mensal de água no reservatório $\left(\mathrm{S}_{(\mathrm{t})}\right)$, conforme dados da Tabela 3.

Tabela 3. Dimensionamento do volume do reservatório com o método de Rippl para o setor de horta.

\begin{tabular}{ccccccccccccc}
\hline Mês & Jan & Fev & Mar & Abr & Mai & Jun & Jul & Ago & Set & Out & Nov & Dez \\
\hline $\mathrm{D}_{(\mathrm{t})}\left(\mathrm{m}^{3}\right)$ & 92 & 74 & 42 & 165 & 283 & 258 & 366 & 419 & 331 & 157 & 42 & 162 \\
$\mathrm{Q}_{(t)}\left(\mathrm{m}^{3}\right)$ & 437 & 328 & 394 & 178 & 60 & 69 & 17 & 8 & 100 & 290 & 419 & 300 \\
$\mathrm{~S}_{(\mathrm{t})}\left(\mathrm{m}^{3}\right)$ & -345 & -254 & -352 & -14 & 223 & 189 & 349 & 411 & 230 & -133 & -377 & -138 \\
\hline
\end{tabular}

Em que: $\mathrm{D}_{(\mathrm{t})}$ - demanda mensal de água da horta; $\mathrm{Q}_{(\mathrm{t})}$ - volume mensal de água da chuva aproveitável; e $\mathrm{S}_{(\mathrm{t})}$ - volume mensal de água no reservatório.

Nos meses de janeiro, fevereiro, março, abril, outubro, novembro e dezembro o volume de água da chuva captado nos telhados é maior que o volume de água necessário para irrigar a horta, portanto nestes meses ocorre excedente de água. A maior demanda de irrigação ocorre na época seca, entre os meses de maio a setembro. Para suprir toda a demanda anual de irrigação do setor de horta, será necessário captar água em $1.922 \mathrm{~m}^{2}$ de área de telhado e armazenar em reservatório com capacidade de $1.402 \mathrm{~m}^{3}$ de água. Já Botari e Prado (2018) verificaram que para irrigar área de gramado de $3.277 \mathrm{~m}^{2}$ no município de Umuarama - PR é necessária área de captação de $500 \mathrm{~m}^{2}$ de telhado e reservatório de $200 \mathrm{~m}^{3}$. A grande diferença de volume de reservatório entre os dois trabalhos ocorreu devido à precipitação em Umuarama, ocorrer em todos os meses do ano e não possuir época seca definida.

\section{CONCLUSÕES}

Para suprir a necessidade hídrica das culturas do setor de horta, será necessário fazer irrigação em todos os meses do ano. A maior demanda de água ocorre na época seca, entre os meses de maio a setembro. Já o período de menores demandas de água necessária para irrigação ocorre entre os meses de outubro e abril, na época das chuvas.

É viável irrigar todo o setor de horta com águas pluviais, captando água da chuva em $1.922 \mathrm{~m}^{2}$ de área de telhado e armazenando a água em reservatório com capacidade de $1.402 \mathrm{~m}^{3}$.

\section{AGRADECIMENTOS}

Os autores agradecem o apoio financeiro do Instituto Federal de Educação, Ciência e 
Tecnologia de Mato Grosso, Campus Campo Novo do Parecis (Edital No 003/2019).

\section{REFERÊNCIAS BIBLIOGRÁFICAS}

AGÊNCIA NACIONAL DE ÁGUAS. Atlas irrigação: uso da água na agricultura irrigada. Brasília, 2017. 86 p.

ALLEN, G. R.; PEREIRA,L.; RAES，D.; SMITH, M. 2006. Estudio FAO Riego y drenaje 56. Evapotranspiración del cultivo: Guías para la determinación de los requerimientos de agua de los cultivos. Serie Cuadernos Técnicos. Roma, Italia. FAO. 298 p.

ASSOCIAÇÃO BRASILEIRA DE NORMAS TÉCNICAS. NBR 15527: Água de chuva Aproveitamento de coberturas em áreas urbanas para fins não potáveis - Requisitos. Rio de Janeiro, 2007. 12 p.

AZEVEDO NETTO, J. M., ARAÚJO, R., FERNANDEZ, M. F., ITO, A. E. Manual de Hidráulica. $8^{\text {a }}$. Ed. São Paulo: Edgard Blucher, 1998. 669 p.

BEZERRA, S. M. da C. et al. Dimensionamento de reservatório para aproveitamento de água de chuva: comparação entre métodos da ABNT NBR 15527:2007 e Decreto Municipal 293/2006 de Curitiba, PR. Ambiente Construído, Porto Alegre, v. 10, n. 4, p. 219-231, 2010. DOI: 10.1590/S167886212010000400015 .

BOTARI, J. C.; PRADO, G. Aproveitamento da água de chuva para irrigação de espaços públicos urbanos abertos: o caso da praça Santos Dumont no município de UMUARAMA - PR. Irriga, Botucatu, v. 23, n. 1 , p. 96-107, 2018. DOI: 10.15809/irriga.2018v23n1p96.

DALCHIAVON, F. C.; MONTANARI, R.; ANDREOTTI, M.; DALLACORT, R.; SOUZA, M. F. P.; Relationship between sunflower productivity and soil's chemical properties by geo-statistical techniques. African Journal of Agricultural Research, v. 10, p. 3525-3532, 2015. DOI: 10.5897 / AJAR2014.9472.

FERERES, E. Papel de la fisiología vegetal en la microirrigación. Recomendaciones para el manejo mejorado. Ponencia en IV Seminario Latinoamericano de Microirrigación. Barquisimeto, Venezuela, 1981. (En prensa).

GHEYI, H. R.; PAZ, V. P. da S.; MEDEIROS, S. de S.; GALVÃO, C. de O. Recursos hídricos em regiões semiáridas: estudos e aplicações. 1. ed. Cruz das Almas, BA. Editora UFRB. 2012. 258 p.

GIL, W. T.; CATAPAN, M. F.; DESCHAMPS, F.; VALLE, P. D. A análise da disponibilidade de recursos hídricos na região metropolitana de Curitiba e a importância das indústrias em buscar fontes alternativas de captação de água. Brazilian Journal of Development, v. 6, n. 3, p. 1374113756, 2020. DOI: 10.34117/bjdv6n3-294.

MANTOVANI, E. C.; BERNARDO, S.; PALARETTI, L. F. Irrigação: princípios e métodos. 3 eds. Atual. Viçosa-MG, Ed. UFV, p. 355, 2009.

MORUZZI, R. B.; OLIVEIRA, S. C.; GARCIA, M. L. A proposal for reservoir volume calculation in rainwater harvesting systems. Journal of Civil Engineering and Architecture, v. 6, n. 6, p.707-714, 2012. DOI: 10.17265 / 1934-7359 / 2012.06.008.

MOSSINI JUNIOR, D., RAMOS, H.C., DALLACORT, R., SILVA, F.S. Distribuição e probabilidade de precipitação para Nova Mutum, Mato Grosso, Brasil. Enciclopédia Biosfera, v. 13, n. 24, p. 435-447, 2016. DOI: 10.18677/EnciBio_2016B_039.

NEU, V.; GUEDES, V. M.; ARAÚJO, M. G. S.; MEYER, L. F. F.; BRITO, I. R; BATISTA, L. M. Água da chuva para consumo humano: estudo de caso na 
Amazônia Oriental. Inclusão Social, v.12, p.183-198, 2018.

NOGUEIRA, C. U.; NOGUEIRA, H. M. C. M.; FANTINEL, A. L.; JAHN, S. L.; PADRÓN, R. A. R. Aproveitamento da água de chuva para lavagem de máquinas agrícolas e veículos: Estudo de caso do Colégio Politécnico da Universidade

Federal de Santa Maria. Revista Eletrônica em Gestão, Educação e Tecnologia Ambiental. Santa Maria, v. 20, n. 1, p. 325333, 2016. DOI: 105902/2236117019415.

TEIXEIRA, C. A.; ZATTONI, G. T.;
NAGALLI, A.; FREIRA, F. B.; TEIXEIRA, S. H. C. Análise de viabilidade técnica e econômica do uso de água de chuva em uma indústria metalmecânica na região metropolitana de Curitiba PR. Gestão \& Produção. São Carlos, v. 23, n. 3, p. 1-11, 2016. DOI: $10.1590 / 0104-530 x 1655-14$.

THORNTHWAITE，C.W.; MATHER，J.R. The water balance. Centerton, $\mathrm{NJ}$ : Drexel Institute of Technology Laboratory of Climatology, 1955. 104p. (Publications in Climatology, vol. VIII, n.1). 